\title{
Neurotrophic Properties of C-Terminal Domain of the Heavy Chain of Tetanus Toxin on Motor Neuron Disease
}

\author{
Mireia Herrando-Grabulosa ${ }^{1,2, *,+}$, Caty Casas ${ }^{3, \pm(\mathbb{D})}$, Kevin Talbot ${ }^{4}\left(\mathbb{D}\right.$ and José Aguilera ${ }^{1,2}$ \\ 1 Institute of Neurosciences, Department of Biochemistry and Molecular Biology, Universitat Autònoma \\ de Barcelona, 08193 Cerdanyola del Vallès, Spain; Jose.Aguilera@uab.cat \\ 2 Centro de Investigación Biomédica en Red sobre Enfermedades Neurodegenerativas (CIBERNED), \\ 28031 Madrid, Spain \\ 3 Institute of Neurosciences, Department of Cell Biology, Physiology and Immunology, Universitat Autònoma \\ de Barcelona, 08193 Cerdanyola del Vallès, Spain; Caty.Casas@uab.cat \\ 4 Nuffield Department of Clinical Neurosciences, University of Oxford, Oxford OX3 9DU, UK; \\ kevin.talbot@ndcn.ox.ac.uk \\ * Correspondence: Mireia.Herrando@uab.cat; Tel.: +34-9-3581-4506 \\ + Present address: Institute of Neurosciences, Department of Cell Biology, Physiology and Immunology, \\ Universitat Autònoma de Barcelona, 08193 Cerdanyola del Vallès, Spain. \\ $\ddagger$ We appreciate the contribution of Caty Casas that unfortunately deceased last July.
}

Received: 16 September 2020; Accepted: 18 October 2020; Published: 21 October 2020

\begin{abstract}
The carboxyl-terminal domain of the heavy chain of tetanus toxin (Hc-TeTx) exerts a neuroprotective effect in neurodegenerative diseases via the activation of signaling pathways related to neurotrophins, and also through inhibiting apoptotic cell death. Here, we demonstrate that Hc-TeTx preserves motoneurons from chronic excitotoxicity in an in vitro model of amyotrophic lateral sclerosis. Furthermore, we found that PI3-K/Akt pathway, but not p21ras/MAPK pathway, is involved in their beneficial effects under chronic excitotoxicity. Moreover, we corroborate the capacity of the Hc-TeTx to be transported retrogradely into the spinal motor neurons and also its capacity to bind to the motoneuron-like cell line NSC-34. These findings suggest a possible therapeutic tool to improve motoneuron preservation in neurodegenerative diseases such as amyotrophic lateral sclerosis.
\end{abstract}

Keywords: carboxyl-terminal domain of the heavy chain of tetanus toxin; neuroprotection; excitotoxicity; spinal muscular atrophy; amyotrophic lateral sclerosis

Key Contribution: The carboxyl-terminal domain of the heavy chain of tetanus toxin (Hc-TeTx) preserves motoneurons from chronic excitotoxicity thought PI3-K/Akt pathway.

\section{Introduction}

Motor neuron diseases (MNDs) are a group of neurodegenerative diseases which, despite having different etiologies and clinical patterns, demonstrate that spinal motoneurons (MNs) are selectively vulnerable. The most common form of MND in adults, amyotrophic lateral sclerosis (ALS), is characterized by combined degeneration of upper and lower MNs, leading to progressive weakness and death from respiratory failure a median of 30 months from symptom onset [1]. Most ALS cases are apparently sporadic (sALS), though about $10 \%$ show familial inheritance (fALS), and specific disease-determining mutations can be identified in up to $15 \%$ cases overall, with more than 50 genes involved. ALS is currently incurable, with available therapies such as riluzole and edaravone only having a trivial impact on disease progression [2,3]. Precision medicine based on approaches to therapy in ALS is urgently needed [4]. 
The carboxyl-terminal domain of the heavy chain of tetanus toxin (Hc-TeTx) is a non-toxic fragment that binds to the cell membrane $[5,6]$. Several studies have demonstrated that Hc-TeTx is able to preferentially bind to MNs [7-11], acting through lipid raft microdomains [12], through gangliosides $[13,14]$ and glycoprotein acceptors $[15,16]$. However, it is still unclear which specific protein receptor mediates binding, although it is known that Hc-TeTx shares trafficking vesicles with some neurotrophins and their receptors in being transported retrogradely to the central nervous system [17]. Moreover, other functions have been described for the non-toxic domain of tetanus toxin. Hc-TeTx can rescue cerebellar granule neurons from apoptotic cell death caused by potassium deprivation and $\mathrm{MPP}^{+}$toxicity $[18,19]$, preserves MNs from acute excitotoxic damage [11], and is an effective neuroprotective agent in several animal models of neurodegeneration [20,21], including a mouse model of ALS [22-24]. Among the molecular mechanisms proposed for Hc-TeTx neuroprotection are the p21ras/MAPK, PI3-K/Akt, and PLC $\gamma / \mathrm{PKC}$ signaling pathways which are downstream of activation of tyrosine kinase receptors (Trks; tropomyosin-related kinases) $[11,18,25,26]$. However, further experiments are needed to clarify the molecular mechanisms underlying the neuroprotective effects of Hc-TeTx. Here, we wanted to demonstrate the neuroprotective mechanisms in a model of chronic excitotoxicity.

\section{Results}

\subsection{Purification and Fluorescence Labeling of Recombinant Protein Hc-TeTx}

In order to obtain a high-quality purification and fluorescence labeling of the recombinant protein Hc-TeTx preparation for further therapeutic treatments and retroaxonal transport analysis, we optimized the purification process of Hc-TeTx by using an FPLC system and cobalt-agarose columns. We determined the eluted protein profile by measuring the absorbance at $280 \mathrm{~nm}$ (Figure 1A). The chromatogram shows a wider peak that represents mixed proteins eluted, and the narrowest peak represents the elution of the Hc-TeTx. The different fractions obtained during the process of purification of the Hc-TeTx protein were treated under reducing conditions and resolved in an electrophoretic gel by loading the same volume of each sample $(15 \mu \mathrm{L})$. A large number of proteins were observed in the wash fractions, whereas in the fractions corresponding to the narrowest peak, the presence of a protein corresponding to a molecular weight of about $50 \mathrm{kDa}$ was identified, such as Hc-TeTx protein. The Hc-TeTx purity of the selected fractions represents around 70\% (Figure 1B). Immunoblot with the anti-histidine antibody allowed detecting the histidine tail of the C-terminal end of the Hc-TeTx protein at $50 \mathrm{kDa}$ (Figure 1C). In order to visualize Hc-TeTx by fluorescence, protein was attached to the small molecule, Alexa Fluor ${ }^{\circledR} 555$. Labeled as Hc-TeTx-Alexa ${ }^{555}$, protein could also be detected at $50 \mathrm{kDa}$ under ultraviolet light (Figure 1D). Dual Color molecular weight markers indicated as M allow detecting a band at $75 \mathrm{kDa}$ and a band at $25 \mathrm{kDa}$ under UV. Overall, these findings show that the recombinant protein Hc-TeTx is produced at high purity and is specifically labeled by fluorescence.

\subsection{Membrane Motoneuron Binding and Retrograde Transport of Recombinant Hc-TeTx Protein}

In order to assess the capacity of the recombinant Hc-TeTx protein to be attached to MNs, we first characterized the ability of the protein to bind to a motor neuron-like cell line, NSC34. We demonstrated that upon addition of labeled Hc-TeTx to the culture, the protein was localized around the NSC-34 cells, suggesting that the incorporation of the fluorescence dye did not modify its capacity to binds to MNs. Moreover, slight labeling can be seen in the soma of NSC-34 cells at 60 min which may be due to the internalization of Hc-TeTx. However, further experiments with specific antibodies against TeTx are needed to confirm this result (Figure 2A). Interestingly, binding to the cell membranes occurs in the first $15 \mathrm{~min}$ after addition to the culture media. These results suggest that Hc-TeTx-Alexa ${ }^{555}$ maintain its properties and it can be attached and may be internalized into MNs. 

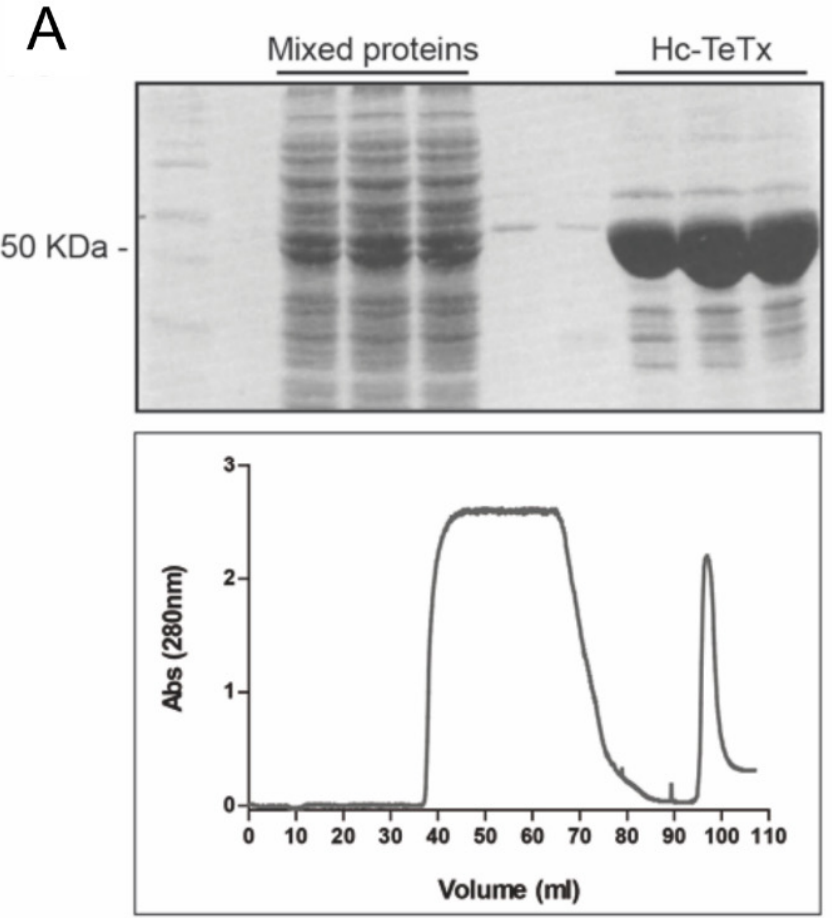
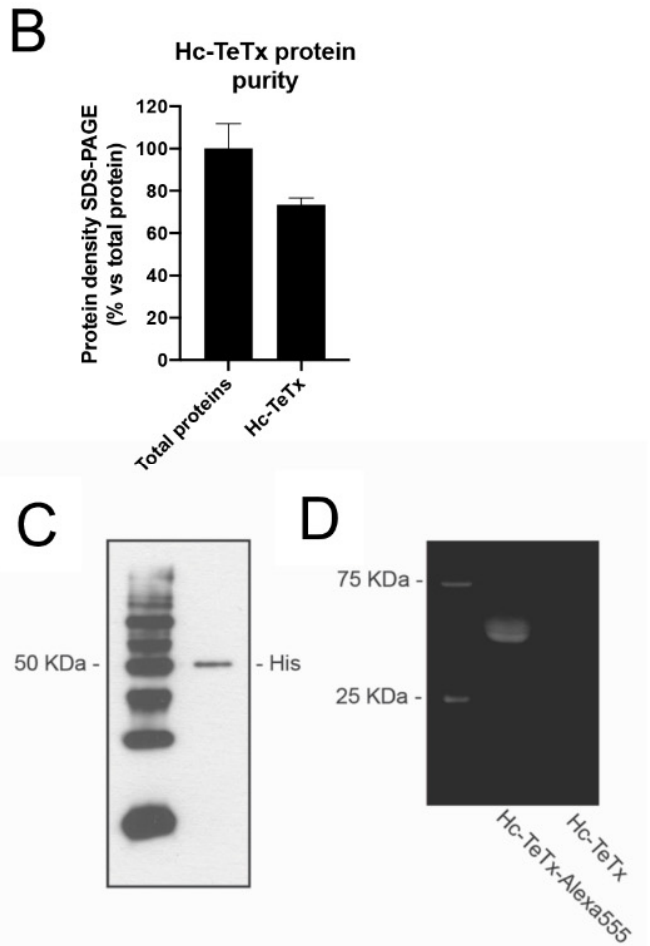

Figure 1. Purification and fluorescence labeling methodology of the carboxyl-terminal domain of the heavy chain of tetanus toxin (Hc-TeTx) protein. (A) Elution profile of the Hc-TeTx protein. Electrophoresis SDS-PAGE stained with comassie blue and fast protein liquid chromatography (FPLC) chromatogram. (B) Densometric quantification of the eluted lanes corresponding to Hc-TeTx fractions. (C) Detection of the Hc-TeTx protein purified using the Western blot technique with the anti-histidine antibody. (D) Labeling detection of the Hc-TeTx protein conjugated to the Alexa Fluor ${ }^{\circledR} 555$ fluorochrome. Histogram and electrophoretic gel representing more than 30 purification processes carried out.

In order to assess the potential for a beneficial role of recombinant Hc-TeTx proteins in MND in vivo models, we wanted to analyze the capacity of our fluorescently labeled purified Hc-TeTx protein to be transported retrogradely into the central nervous system. Hc-TeTx were administered by focal injection into the right tibialis muscle (TA) of wild type mice at $50 \mathrm{ng} / \mathrm{Kg}$ to ensure enough protein would be transported into the ventral horn MN soma. At $24 \mathrm{~h}$ post-injection, Hc-TeTx can be localized at the neuromuscular junction (NMJ) of the right TA muscle, co-labeled with bungarotoxin (Figure 2B). Moreover, we demonstrated that recombinant protein Hc-TeTx cannot diffuse to other muscles, such as neighboring gastrocnemius (GM), or muscles more remote from the injection site, such as the left TA and GM (Figure 2C), and it is inside the NMJ (Figure 2D). At $24 \mathrm{~h}$, the recombinant protein injected into the right TA muscle can be detected in the corresponding ventral horn motor neuron pool innervating TA muscle (Figure 2E). However, further experiments with specific antibodies against TeTx are needed to confirm this result. These findings suggest that the intramuscular injection of recombinant Hc-TeTx protein can be internalized in the NMJ and transported retrogradely to the spinal cord and is an effective method of delivery of a potentially neuroprotective agent with high affinity and specificity for spinal MNs for future studies in vivo. 
A

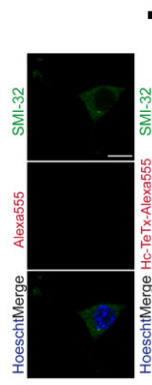

B

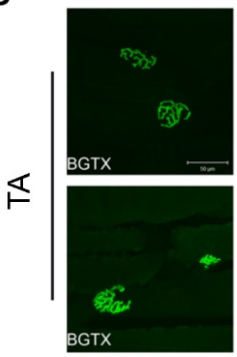

Internalization time
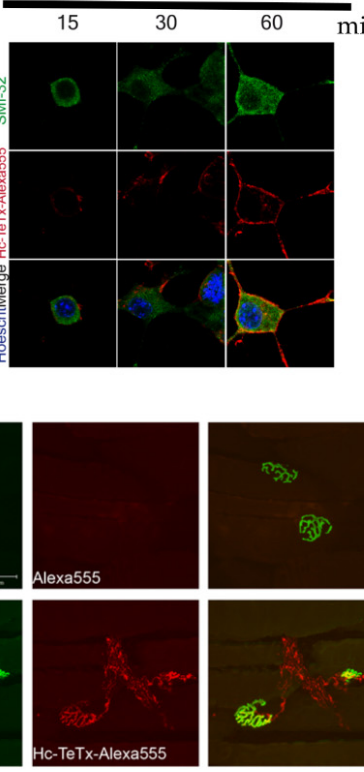

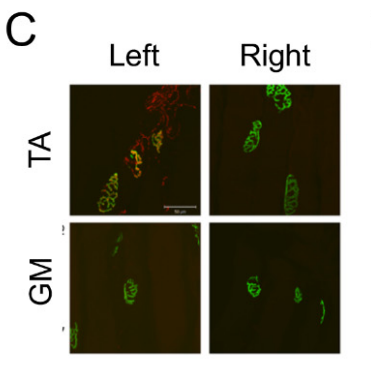

$\mathrm{D}$

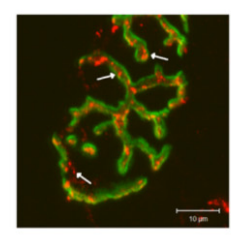

E

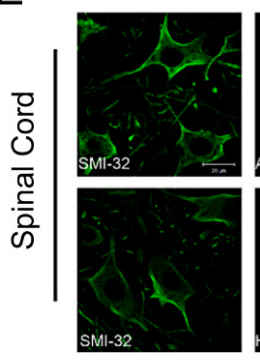

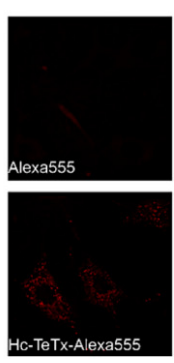

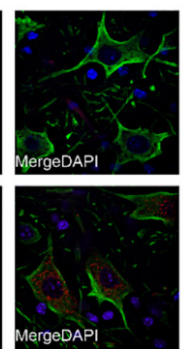

Figure 2. Internalization and retrograde transport of Hc-TeTx-Alexa ${ }^{555}$ protein on motoneurons. (A) Time-course of Hc-TeTx-Alexa ${ }^{555}$ internalization in the NSC-34 cells, a motoneuron-like cell line. Controls performed by adding the same amount of fluorochrome did not show fluorescence at the indicated times. The bar represents $10 \mu \mathrm{m}$. (B) The Hc-TeTx-Alexa ${ }^{555}$ (red) protein is detected in motor nerve endings identified with $\alpha$-bungarotoxin (BGTX) (green) $24 \mathrm{~h}$ after a single intramuscular injection into the tibialis anterior (TA) muscle. The injection with the same amount of fluorocrom (Alexa ${ }^{555}$ ) does not emit fluorescence in the neuromuscular junction (NMJ). (C) The Hc-TeTx-Alexa ${ }^{555}$ protein can be detected in the injected muscle but does not spread to other muscles such as gastrocnemius muscles (GM) of the same leg (Left leg) or in the TA muscles and GM of the right leg, where there is no presence of Hc-TeTx-Alexa ${ }^{555}$. (D) At $24 \mathrm{~h}$ the Hc-TeTx-Alexa ${ }^{555}$ protein is located inside the NMJ without colocalizing with AChR receptors. The arrows indicate the formation of clusters inside the NMJ and presynaptic endings. Representative images of 3 independent experiments by condition. (E) Visualization of the Hc-TeTx-Alexa ${ }^{555}$ protein in the spinal cord at $24 \mathrm{~h}$ after intramuscular injection. The Hc-TeTx-Alexa ${ }^{555}$ protein (red) was visualized inside the soma of the motor neurons (green), which were marked with anti-SMI-32. The markup of Hc-TeTx-Alexa ${ }^{555}$ followed a vesicular pattern. The control injection with the same amount of fluorocrom (Alexa Fluor ${ }^{\circledR 555}$ ) did not emit fluorescence in the spinal cord.

\subsection{Motor Neuron Preservation under Chronic Excitotoxicity}

In order to assess the neuroprotective capacity of recombinant Hc-TeTx protein, we first reproduced a well-established in vitro model of progressive chronic glutamate excitotoxicity by the addition of DL-threo- $\beta$-hydroxyaspartic acid (THA), an inhibitor of glutamate transport, using organotypic spinal cord slice cultures. After 7 days in vitro (DIV), we administered THA, accompanied by either vehicle or recombinant Hc-TeTx protein, at $10 \mathrm{nM}$, according to previous studies [13]. THA reduces MN numbers to about $60 \%$ in comparison to the control, but the addition of Hc-TeTx allows protecting the number of MNs, reaching $80 \%$ of $\mathrm{MN}$ preservation. In contrast, $\mathrm{MN}$ counting revealed that $\mathrm{Hc}-\mathrm{TeTx}$ cannot enhance the number of MNs under basal culture conditions (Figure 3). Given that chronic excitotoxicity mediated by glutamate is implicated in the pathogenesis of ALS, these results indicate that Hc-TeTx protein has the potential to act as a neuroprotective factor in ALS. 

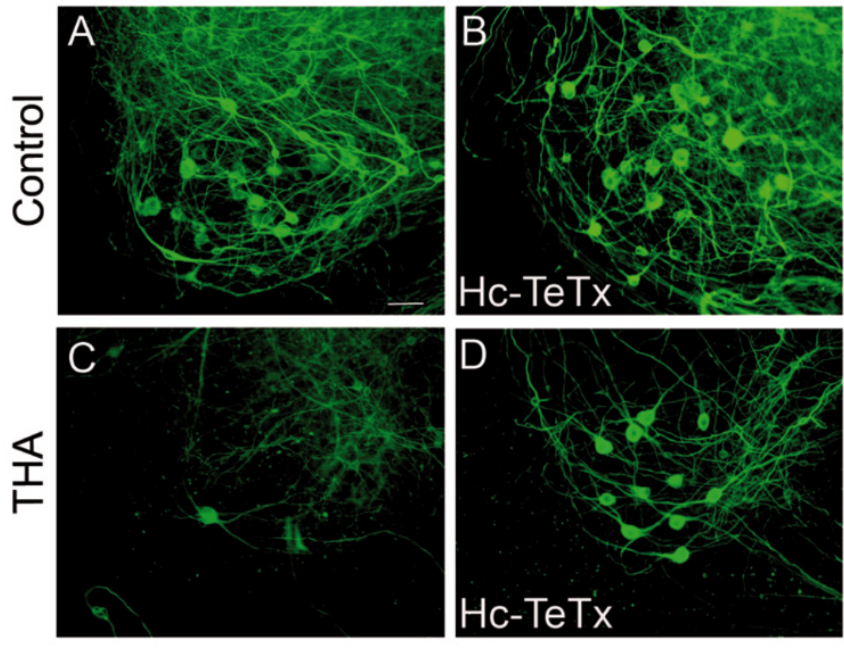

E

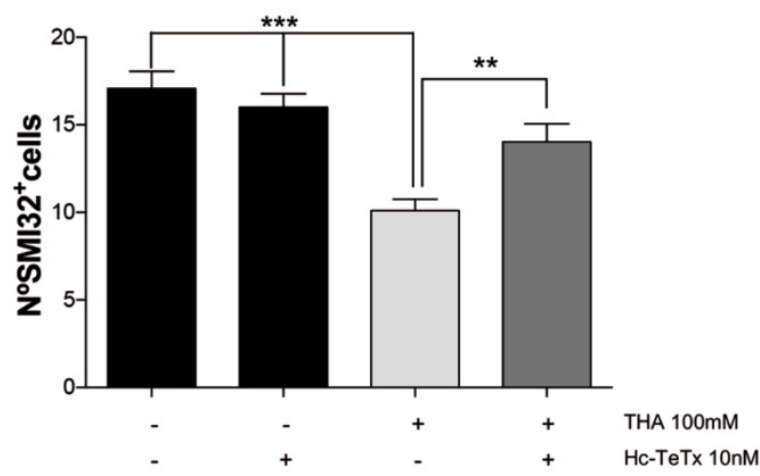

Figure 3. Evaluation of the neuroprotective effect of the Hc-TeTx protein against chronic excitotoxic damage caused by the addition of DL-threo- $\beta$-hydroxyaspartic acid (THA) (100 $\mu \mathrm{M})$ in spinal cord organotypic cultures. Motoneurons (MNs) located in the ventral horn of the spinal cord were visualized by immunofluorescence with anti-SMI-32. The addition of THA $(100 \mu \mathrm{M})$ at 7, 14, and 21 days in vitro (DIV) caused a loss of MNs (C) with respect to the control (A). The treatment with Hc-TeTx $(10 \mathrm{nM})$ under conditions of chronic excitotoxicity allowed the viability of the MNs (D). There were no differences in the viability of the MNs with treatment with Hc-TeTx under controlled conditions (B). (E) Representative micrographs of the counting of positive SMI-32 cells in the ventral area of the spinal cord. The values are shown according to \pm SEM with a minimum of 15 sections per treatment. On ${ }^{* *} p<0.001$ and ${ }^{* *} p<0.01$ with respect to THA $(100 \mu \mathrm{M})$ according to an ANOVA analysis with the statistical test of Bonferroni. The bar represents $50 \mu \mathrm{m}$.

\subsection{Signaling Cascade Induced by Hc-TeTx under Chronic Excitotoxicity}

Previously, we demonstrated that neuroprotective effects of recombinant Hc-TeTx protein after acute excitotoxicity act via p21ras/MAPK and PI3-K/Akt signaling pathways [11]. Here, we found that the specific inhibitor for PI3-Kinases (LY294002) blocks the neuroprotective effect of Hc-TeTx under chronic excitotoxicity, but the inhibition of MEK1 (PD98059) did not block its beneficial effect (Figure 4A,B). Treatment of spinal cord cultures with both inhibitors under basal and excitotoxic conditions alone had no effect on MN survival. These findings suggest that recombinant Hc-TeTx protein exerts a neuroprotective role specifically through the PI3-K/Akt signaling pathway in an in vitro organotypic model of chronic excitotoxicity, which may have implications for targeting therapy in ALS. 

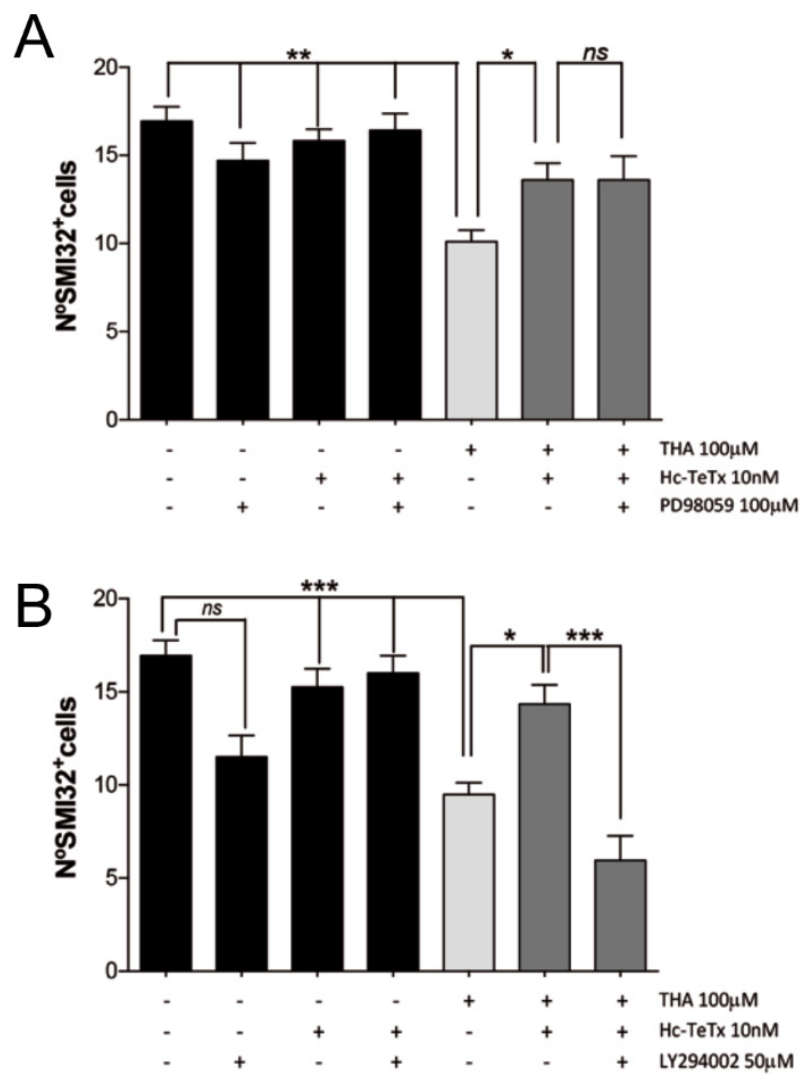

Figure 4. Hc-TeTx activates PI3-K/Akt signaling pathway in a chronic excitotoxicity model caused by the addition of DL-threo- $\beta$-hydroxyaspartic acid (THA). Organotypic cultures were treated with the glutamate transporter inhibitor (THA) at $100 \mu \mathrm{M}$ and the Hc-TeTx protein at $10 \mathrm{nM}$. (A) Number of SMI- $32^{+}$cells countered after addition of the MEK inhibitor, PD98059 at 100 $\mathrm{MM}$. (B) Number of SMI-32 ${ }^{+}$

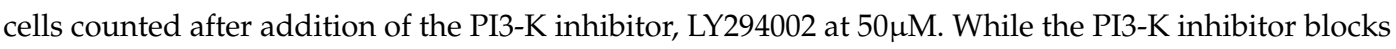
the neuroprotective effect of Hc-TeTx protein, in the face of chronic excitotoxicity, the MEK inhibitor does not impede this ability. The values are shown according to \pm SEM with a minimum of 15 sections per treatment. On ${ }^{* * *} p<0.001,{ }^{* *} p<0.01$ and ${ }^{*} p<0.05$ with respect to THA $(100 \mu \mathrm{M})$ according to an ANOVA analysis with the statistical test of Bonferroni.

\section{Discussion}

The preservation of MNs from cell death is a relevant goal in MNDs. In ALS, lack of early biomarkers of disease activity is a barrier to initiating treatment early in disease, which is likely to be the most therapeutically tractable phase. Several previous studies have suggested neuroprotective effects of Hc-TeTx in a number of neurodegenerative diseases [20,21]. Moreover, recent studies have demonstrated that Hc-TeTx promotes locomotor recovery and motor neuron survival after spinal cord injury in rats, decreasing apoptotic markers of cell death and modulating autophagy [11,27]. We have previously demonstrated that Hc-TeTx can promote the preservation of MNs under conditions of acute excitotoxic damage [11].

Here, we demonstrated that Hc-TeTx can be easily produced at high purity. Conjugation with fluorochromes facilitates easier tracking. In order to evaluate the potential neuroprotective effect of Hc-TeTx protein in ALS, we used an organotypic spinal cord model based on the inhibition of glutamate carriers by the addition of THA $(100 \mu \mathrm{M})$ [28]. This ex vivo system is viewed as an established model of progressive excitotoxicity (slow excitotoxicity) and a very useful tool for the study of motor neuron degeneration. The origin of sporadic forms of ALS remains unclear, but it is now recognized to be a multisystemic degeneration, involving multiple pathophysiological molecular 
processes, including excitotoxicity [4,29]. In patients with ALS, high levels of glutamate have been detected in the cerebrospinal fluid (CSF), as well as low expression levels of the EAAT2 glutamate transporter, although whether this is the cause of MN death or a consequence remains debated [30-32]. Glutamate carriers play a very important role in the buffering cells due to the toxic effects of excess extracellular glutamate [33]. There is controversy around how chronically damaged motoneurons die from excitotoxicity. During the degeneration of spinal MNs, apoptotic, necrotic, and autophagic processes have been implicated, though the final common pathway of death of MNs may be mainly due to apoptosis $[34,35]$.

The beneficial role of Hc-TeTx in an in vitro model of glutamate excitotoxicity is due specifically to the PI3-K/Akt signaling pathway. Hc-TeTx protein can also modulate autophagic pathways via PI3-K/Akt [11]. Further experiments are needed to explore the capacity of recombinant Hc-TeTx protein to preserve motoneurons and motor function in a mouse model of ALS such as SOD1G93A.

In the current study, we additionally wanted to highlight the capacity of fluorescently detecting the Hc-TeTx recombinant protein to reveal its high capacity to be internalized in MNs and to be transported retrogradely, supporting the potential of further in vivo experiments. Results from NSC34 cells reinforce the rapid capacity of Hc-TeTx to bind to MNs. Moreover, results in mice suggest that $\mathrm{Hc}-\mathrm{TeTx}$ can be transported retrogradely in just $24 \mathrm{~h}$.

In summary, Hc-TeTx recombinant protein preserves MNs from chronic degenerative process, specifically through the PI3-K/Akt signaling pathway. The capacity of Hc-TeTx to be transported retrogradely suggests that further work should focus on studies to elucidate the protein partners by which Hc-TeTx is transported at a faster rate than other retrotracers.

\section{Materials and Methods}

\subsection{Recombinant Hc-TeTx Protein Purification}

Escherichia coli BL21 cells were transformed by pQE3-Hc-TeTx-Hisx6 plasmid and cells were grown in Luria Bertani medium containing $100 \mu \mathrm{g} / \mathrm{mL}$ ampicillin. Protein expression was induced by the addition of $0.4 \mathrm{mM}$ isopropyl $\beta \mathrm{D}$ thiogalactoside (IPTG). After $3 \mathrm{~h}$, cells were pelleted by centrifugation at $4000 \times g$ for $20 \mathrm{~min}$ at $4{ }^{\circ} \mathrm{C}$, resuspended in lysis buffer $\left(50 \mathrm{mM} \mathrm{NaH}_{2} \mathrm{PO}_{4}, 300 \mathrm{mM}\right.$ $\mathrm{NaCl}$ and $1 \%$ Triton $\times 100 ; \mathrm{pH} 8$ ) and sonicated on ice for six 30 s periods. The suspension was centrifuged at $30,000 \times g$ for $30 \mathrm{~min}$ at $4{ }^{\circ} \mathrm{C}$. The clear supernatant, which contains the His-tagged protein, was purified by cobalt affinity chromatography. Mixed proteins were injected in a fast protein liquid chromatography (FPLC), which contains a cobalt-agarose resin (TALON Metal Affinity resin, Clontech Laboratories; Palo Alto, CA, USA), previously equilibrated (50 $\mathrm{mM} \mathrm{NaH}_{2} \mathrm{PO}_{4}$ and $300 \mathrm{mM}$ $\mathrm{NaCl} ; \mathrm{pH} 7)$. The proteins, without His-Tags, were eluted by washing the resin with elution buffer (50 $\mathrm{mM} \mathrm{NaH}_{2} \mathrm{PO}_{4}$ and $300 \mathrm{mM} \mathrm{NaCl} ; \mathrm{pH}$ 7). Hc-TeTx contains six histidine residues and is retained in the resin forming a Co-complex. Hc-TeTx was eluted with the elution buffer $\left(50 \mathrm{mM} \mathrm{NaH} \mathrm{PO}_{4}\right.$, $300 \mathrm{mM} \mathrm{NaCl}$ and $150 \mathrm{mM}$ Imidazole; $\mathrm{pH}$ 7). Fractions collected were $0.5 \mathrm{~mL}$ in volume. The elution process can be followed with an FPLC system that constantly measures the absorbance at $280 \mathrm{~nm}$. Protein was separated by sodium dodecyl sulfate-polyacrylamide gel electrophoresis (SDS-PAGE) at 12\%. Gel was stained with GelCode Blue Stain Reagent (Pierce Chemical Co.; Rockford, IL, USA) and those fractions containing purified Hc-TeTx protein were dialyzed $\left(40 \mathrm{mM} \mathrm{Na}_{2} \mathrm{HPO}_{4}, 10 \mathrm{mM} \mathrm{NaH} \mathrm{PO}_{4}\right.$ and $150 \mathrm{mM} \mathrm{NaCl} ; \mathrm{pH} 7.4$ ) overnight at $4{ }^{\circ} \mathrm{C}$ and for $2 \mathrm{~h}$ with new buffer. Protein concentrations were determined using the bicinchoninic acid assay (BCA, Pierce Chemical Co.; Rockford, IL, USA) and lyophilized. The Hc-TeTx was stored in aliquots at $-20^{\circ} \mathrm{C}$. Alexa Fluor ${ }^{\circledR} 555$ was attached to Hc-TeTx following the manufacturer's protocol from Alexa Fluor ${ }^{\circledR} 555$ Labeling Kit (Invitrogen; Carlsbad, CA, USA) and stored at $-20^{\circ} \mathrm{C}$. 


\subsection{Hc-TeTx Retrogade Transport}

Single intramuscular injection of Hc-TeTx-Alexa ${ }^{555}$ at $50 \mathrm{ng} / \mathrm{kg}$ was performed into the right tibialis anterior muscle in wild-type (WT) adult mice to assess the capacity of the conjugated protein to be retrogradely transported. After $24 \mathrm{~h}$, right and left tibialis and gastrocnemius muscles were collected and fixed in PFA $4 \%$. Then, tissue was cryopreserved with sucrose at $4{ }^{\circ} \mathrm{C}$.

\subsection{NSC-34 Cell Culture}

NSC-34 cells were grown in coated poly-D-lysine 24-well plates with $1 \mathrm{~mL}$ Glutamax with $10 \%$ $(v / v)$ FBS. Cells were plated at $2.5 \times 10^{5}$ cells/well for experiments. After $48 \mathrm{~h}$, the medium was replaced with Dubelcco Modified Eagle Medium (DMEM) plus 10\% (v/v) FBS.

\subsection{Spinal Cord Organotypic Culture}

Organotypic spinal cord cultures were prepared from lumbar spinal cords of 8-day-old Sprague-Dawley rat pups (P8), as previously described [13,29,30]. After euthanasia, the spinal cord was collected under sterile conditions and placed in ice-cold high glucose-containing $(6.4 \mathrm{mg} / \mathrm{mL})$ Gey's Balanced Salt Solution (GBSS) (Sigma-Aldrich Corp.; St. Louis, MO, USA). Meninges and roots were removed, and the spinal cord was transversely sectioned in $350 \mu \mathrm{m}$ slices with a McIlwain Tissue Chopper (The Mickle Laboratory Engineering Co.; Surrey, UK). Five sections were carefully transferred on Millicell-CM porous membranes $(0.4 \mu \mathrm{m})$ (Millipore; Billerica, MA, USA) into a 6-well plate containing $1 \mathrm{~m}$ : of incubation medium (50\% minimal essential medium (MEM), $25 \mathrm{mM}$ Hepes, $25 \%$ heat-inactivated horse serum, $2 \mathrm{mM}$ glutamine and 25\% Hank's Balanced Salt Solution (HBSS) supplemented with $25.6 \mathrm{mg} / \mathrm{mL}$ glucose; $\mathrm{pH} 7.2$ ). Cultures were incubated at $37^{\circ} \mathrm{C}$ in a $5 \% \mathrm{CO}_{2} / 95 \%$ air humidified environment. Cultures were left to stabilize for one week, and after this, the medium was changed twice per week until 28 days in vitro (DIV). Under these conditions, the MN population reaches a steady number from one to four weeks, as previously described [30]. All procedures involving animals were approved by the Ethics Committee of Universitat Autònoma de Barcelona and followed the European Communities Council Directive 86/609/EEC. After 7 days of the explant culture, chronic glutamate excitotoxicity will be induced by DL-threo- $\beta$-hydroxyaspartic acid (THA) at $100 \mu \mathrm{M}, 1$ to 4 weeks, a potent glutamate transporter inhibitor, which is known to produce a dose-dependent sustained elevation of glutamate levels, causing degeneration of motor neurons [28]. The neuroprotective effect of Hc-TeTx at $10 \mathrm{nM}$ will be assessed by its co-addition to the culture with THA and renewal of each medium change.

\subsection{Motor Neuron Counting}

We identified MNs in the slices by immunostaining with SMI-32 antibody and according to their localization in the ventral horn of lumbar sections. All thoracic and sacral sections were excluded. We blindly counted MNs meeting these criteria in each spinal cord section. To count SMI-32 positive cells in the ventral horn of organotypic spinal cord slices, we used Z-stack confocal series.

\subsection{Immunofluorescence}

Spinal cord slices were fixed with $4 \%$ paraformaldehyde at room temperature for $1 \mathrm{~h}$. Slices were then washed twice with phosphate-buffered saline (PBS) for $15 \mathrm{~min}$, blocked with 5\% normal horse serum and $0.2 \%$ Triton-X100 in PBS, and incubated overnight at $4{ }^{\circ} \mathrm{C}$ with antibody against mouse anti-neurofilament heavy-chain (NF-H) (SMI-32, 1:1000) (Sternberger Monoclonals Inc.; Baltimore, MD, USA). Cultures were then thoroughly washed in PBS with 0.2\% Tween-20 (PBS-T) and incubated with appropriate secondary antibody Alexa Fluor ${ }^{\circledR} 488$ goat anti-mouse $\operatorname{IgG}(1: 1000)$ diluted in blocking buffer for $1 \mathrm{~h}$ at room temperature. Then, slices were washed two times with PBS-T, incubated for 20 min with DAPI (4',6-Diamidino-2-phenylindole), diluted in PBS, and washed several times. Finally, slices were mounted in Superfrost ${ }^{\circledR}$ Plus slides (Thermo Fisher Scientific; Waltham, MA, USA) 
with Fluoromount-G mounting medium (SouthernBiotech; Birmingham, AL, USA) and fluorescence was visualized under an epifluorescence microscope (Nikon Eclipse 90i, Nikon Instruments Inc.; Melville, NY, USA) or a confocal microscope (Leica TSC SP5, Leica Microsystems; Deerfield, IL, USA). A minimum of 15 sections were used for $\mathrm{MN}$ counting for each experimental condition.

\subsection{Statistical Analysis}

Statistical significance was determined by one-way ANOVA, followed by Bonferroni's post hoc test. Differences were considered to be significant if $p<0.05$.

Author Contributions: M.H.-G., C.C., K.T. and J.A. supervised the study and designed experiments; M.H.-G. performed experiments and analysed data; M.H.-G. wrote the manuscript; C.C., K.T. and J.A. made manuscript revisions. All authors have read and agreed to the published version of the manuscript.

Funding: This research was funded by SAF2016-80027-R and PID2019-106615RB-I00 from the Ministerio de Ciencia e Innovación from the Spanish Government.

Acknowledgments: The excellent technical assistance of Cristina Gutierrez in cell culture is gratefully acknowledged. To Xavier Navarro for his support on part of the work.

Conflicts of Interest: The authors declare no conflict of interest.

\section{References}

1. Wijesekera, L.C.; Leigh, P.N. Amyotrophic lateral sclerosis. Orphanet J. Rare Dis. 2009, 4, 3. [CrossRef] [PubMed]

2. Ludolph, A.C.; Jesse, S. Evidence-based drug treatment in amyotrophic lateral sclerosis and upcoming clinical trials. Ther. Adv. Neurol. Disord. 2009, 2, 319-326. [CrossRef] [PubMed]

3. Abe, K.; Aoki, M.; Tsuji, S.; Itoyama, Y.; Sobue, G.; Togo, M.; Hamada, C.; Tanaka, M.; Akimoto, M.; Nakamura, K.; et al. Safety and efficacy of edaravone in well defined patients with amyotrophic lateral sclerosis: A randomised, double-blind, placebo-controlled trial. Lancet Neurol. 2017, 16, 505-512. [CrossRef]

4. Talbot, K.; Feneberg, E.; Scaber, J.; Thompson, A.G.; Turner, M.R. Amyotrophic lateral sclerosis: The complex path to precision medicine. J. Neurol. 2018, 265, 2454-2462. [CrossRef] [PubMed]

5. Bizzini, B.; Stoeckel, K.; Schwab, M. An antigenic polypeptide fragment isolated from tetanus toxin: Chemical characterization, binding to gangliosides and retrograde axonal transport in various neuron systems. J. Neurochem. 1977, 28, 529-542. [CrossRef]

6. Manning, K.A.; Erichsen, J.T.; Evinger, C. Retrograde transneuronal transport properties of fragment $\mathrm{C}$ of tetanus toxin. Neuroscience 1990, 34, 251-263. [CrossRef]

7. Price, D.L.; Griffin, J.; Young, A.; Peck, K.; Stocks, A. Tetanus toxin: Direct evidence for retrograde intraaxonal transport. Science 1975, 30, 945-947. [CrossRef]

8. Lalli, G.; Schiavo, G. Analysis of retrograde transport in motor neurons reveals common endocytic carriers for tetanus toxin and neurotrophin receptor p75NTR. J. Cell Biol. 2002, 156, 233-239. [CrossRef]

9. Lalli, G.; Bohnert, S.; Deinhardt, K.; Verastegui, C.; Schiavo, G. The journey of tetanus and botulinum neurotoxins in neurons. Trends Microbiol. 2003, 11, 431-437. [CrossRef]

10. Roux, S.; Colasante, C.; Saint Cloment, C.; Barbier, J.; Curie, T.; Girard, E.; Molgó, J.; Brûlet, P. Internalization of a GFP-tetanus toxin C-terminal fragment fusion protein at mature mouse neuromuscular junctions. Mol. Cell Neurosci. 2005, 30, 572-582. [CrossRef]

11. Herrando-Grabulosa, M.; Casas, C.; Aguilera, J. The C-terminal domain of tetanus toxin protects motor neurons against acute excitotoxic damage on spinal cord organotypic cultures. J. Neurochem. 2013, 124, 36-44. [CrossRef] [PubMed]

12. Herreros, J.; Ng, T.; Schiavo, G. Lipid rafts act as specialized domains for tetanus toxin binding and internalization into neurons. Mol. Biol. Cell 2001, 12, 2947-2960. [CrossRef] [PubMed]

13. Halpern, J.L.; Loftus, A. Characterization of the receptor-binding domain of tetanus toxin. J. Biol. Chem. 1993, 268, 11188-11192. [PubMed]

14. Benson, M.A.; Fu, Z.; Kim, J.J.; Baldwin, M.R. Unique ganglioside recognition strategies for clostridial neurotoxins. J. Biol. Chem. 2011, 286, 34015-34022. [CrossRef] 
15. Yavin, E.; Nathan, A. Tetanus toxin receptors on nerve cells contain a trypsin-sensitive component. Eur. J. Biochem. 1986, 154, 403-407. [CrossRef] [PubMed]

16. Herreros, J.; Lalli, G.; Montecucco, C.; Schiavo, G. Tetanus toxin fragment $\mathrm{C}$ binds to a protein present in neuronal cell lines and motor neurons. J. Neurochem. 2000, 74, 1941-1950. [CrossRef]

17. Deinhardt, K.; Salinas, S.; Verastegui, C.; Watson, R.; Worth, D.; Hanrahan, S.; Bucci, C.; Schiavo, G. Rab5 and Rab7 control endocytic sorting along the axonal retrograde transport pathway. Neuron 2006, 52, 293-305. [CrossRef] [PubMed]

18. Chaib-Oukadour, I.; Gil, C.; Aguilera, J. The C-terminal domain of the heavy chain of tetanus toxin rescues cerebellar granule neurones from apoptotic death: Involvement of phosphatidylinositol 3-kinase and mitogen-activated protein kinase pathways. J. Neurochem. 2004, 90, 1227-1236. [CrossRef]

19. Chaïb-Oukadour, I.; Gil, C.; Rodríguez-Alvarez, J.; Ortega, A.; Aguilera, J. Tetanus toxin H(C) fragment reduces neuronal MPP+ toxicity. Mol. Cell Neurosci. 2009, 41, 297-303. [CrossRef]

20. Mendieta, L.; Venegas, B.; Moreno, N.; Patricio, A.; Martínez, I.; Aguilera, J.; Limon, I.D. The carboxyl-terminal domain of the heavy chain of tetanus toxin prevents dopaminergic degeneration and improves motor behavior in rats with striatal MPP(+)-lesions. Neurosci. Res. 2009, 65, 98-106. [CrossRef]

21. Oliván, S.; Calvo, A.C.; Rando, A.; Herrando-Grabulosa, M.; Manzano, R.; Zaragoza, P.; Tizzano, E.F.; Aquilera, J.; Osta, R. Neuroprotective Effect of Non-viral Gene Therapy Treatment Based on Tetanus Toxin C-fragment in a Severe Mouse Model of Spinal Muscular Atrophy. Front. Mol. Neurosci. 2016, 9, 76. [CrossRef] [PubMed]

22. Calvo, A.C.; Moreno-Igoa, M.; Mancuso, R.; Manzano, R.; Oliván, S.; Muñoz, M.J.; Penas, C.; Zaragoza, P.; Navarro, X.; Osta, R. Lack of a synergistic effect of a non-viral ALS gene therapy based on BDNF and a TTC fusion molecule. Orphanet J. Rare Dis. 2011, 6, 10. [CrossRef] [PubMed]

23. Moreno-Igoa, M.; Calvo, A.C.; Penas, C.; Manzano, R.; Oliván, S.; Muñoz, M.J.; Mancuso, R.; Zaragoza, P.; Aguilera, J.; Navarro, X.; et al. Fragment $C$ of tetanus toxin, more than a carrier. Novel perspectives in non-viral ALS gene therapy. J. Mol. Med. 2010, 88, 297-308. [CrossRef] [PubMed]

24. Moreno-Igoa, M.; Calvo, A.C.; Ciriza, J.; Muñoz, M.J.; Zaragoza, P.; Osta, R. Non-viral gene delivery of the GDNF, either alone or fused to the C-fragment of tetanus toxin protein, prolongs survival in a mouse ALS model. Restor. Neurol. Neurosci. 2012, 30, 69-80. [CrossRef]

25. Gil, C.; Chaib-Oukadour, I.; Blasi, J.; Aguilera, J. HC fragment (C-terminal portion of the heavy chain) of tetanus toxin activates protein kinase $C$ isoforms and phosphoproteins involved in signal transduction. Biochem. J. 2001, 356 Pt 1, 97-103. [CrossRef]

26. Gil, C.; Chaib-Oukadour, I.; Aguilera, J. C-terminal fragment of tetanus toxin heavy chain activates Akt and MEK/ERK signalling pathways in a Trk receptor-dependent manner in cultured cortical neurons. Biochem. J. 2003, 373 Pt 2, 613-620. [CrossRef]

27. Sozbilen, M.C.; Ozturk, M.; Kaftan, G.; Dagci, T.; Ozyalcin, H.; Armagan, G. Neuroprotective Effects of C-terminal Domain of Tetanus Toxin on Rat Brain Against Motorneuron Damages After Experimental Spinal Cord Injury. Spine 2018, 43, E327-E333. [CrossRef] [PubMed]

28. Rothstein, J.D.; Jin, L.I.N.; Dykes-Hoberg, M.; Kuncl, R.W. Chronic inhibition of glutamate uptake produces a model of slow neurotoxicity. Proc. Natl. Acad. Sci. USA 1993, 90, 6591-6595. [CrossRef]

29. Ferraiuolo, L.; Kirby, J.; Grierson, A.J.; Sendtner, M.; Shaw, P.J. Molecular pathways of motor neuron injury in amyotrophic lateral sclerosis. Nat. Rev. Neurol. 2011, 7, 616-630. [CrossRef]

30. Rothstein, J.D.; Martin, L.J.; Kuncl, R.W. Decreased glutamate transport by the brain and spinal cord in amyotrophic lateral sclerosis. N. Engl. J. Med. 1992, 326, 1464-1468. [CrossRef] [PubMed]

31. Rothstein, J.D.; Van Kammen, M.; Levey, A.I.; Martin, L.J.; Kuncl, R.W. Selective loss of glial glutamate transporter GLT-1 in amyotrophic lateral sclerosis. Ann. Neurol. 1995, 38, 73-84. [CrossRef] [PubMed]

32. Fray, A.E.; Ince, P.G.; Banner, S.J.; Milton, I.D.; Usher, P.A.; Cookson, M.R.; Shaw, P.J. The expression of the glial glutamate transporter protein EAAT2 in motor neuron disease: An immunohistochemical study. Eur. J. Neurosci. 1998, 10, 2481-2489. [CrossRef] [PubMed]

33. Foran, E.; Trotti, D. Glutamate transporters and the excitotoxic path to motor neuron degeneration in amyotrophic lateral sclerosis. Antioxid. Redox Signal. 2009, 11, 1587-1602. [CrossRef] [PubMed] 
34. Matyja, E.; Nagańska, E.; Taraszewska, A.; Rafatowska, J. The mode of spinal motor neurons degeneration in a model of slow glutamate excitotoxicity in vitro. Folia Neuropathol. 2005, 43, 7-13.

35. Matyja, E.; Taraszewska, A.; Nagańska, E.; Rafałowska, J. Autophagic degeneration of motor neurons in a model of slow glutamate excitotoxicity in vitro. Ultrastruct. Pathol. 2005, 29, 331-339. [CrossRef] [PubMed]

Publisher's Note: MDPI stays neutral with regard to jurisdictional claims in published maps and institutional affiliations.

(C) 2020 by the authors. Licensee MDPI, Basel, Switzerland. This article is an open access article distributed under the terms and conditions of the Creative Commons Attribution (CC BY) license (http://creativecommons.org/licenses/by/4.0/). 